\title{
A Potencialidade das Macroalgas na Remoção de Elementos Terras Raras de Águas Contaminadas
}

\author{
Filipe L. S. Monteiro* \\ Jéssica Jacinto \\ João Colónia \\ Nicole Ferreira \\ Thainara Viana \\ João Pinto \\ Daniela Tavares \\ Carlos Vale \\ Eduarda Pereira \\ Bruno Henriques
}

\author{
Potential of Macroalgae to Remove Rare \\ Earth Elements from Contaminated Waters. \\ The European Union has classified rare earth \\ elements (REE) as critical raw materials of \\ high economic value and commercial interest, \\ resulting from the difficulty and high costs \\ to obtain them, while having a wide field \\ of applications. This work evaluates the use \\ of different live macroalgae to remove and \\ concentrate REE from waters where they are \\ present. The potential use of live macroalgae \\ was evaluated through kinetic tests in \\ mono- and multi-contaminated solutions, \\ incorporating REE Cerium (Ce), Europium (Eu), \\ Yttrium (Y), Lanthanum (La) and Neodymium \\ (Nd) in saline water, and starting from an \\ initial concentration of $500 \mu \mathrm{g} \mathrm{L}^{-1}$, to which \\ approximately 450-500 $\mathrm{mg} \mathrm{L}^{-1}$ in dry weight \\ of live macroalgae Gracilaria gracilis, Fucus \\ vesiculosus and Ulva lactuca were added. The \\ removal rates achieved by the red seaweed \\ G. gracilis stood out, with values between \\ 60 and $99 \%$ for all the REE in solution. The \\ multi-contaminated solutions showed removal \\ efficiencies higher than those obtained with \\ the mono-contaminated, further proving \\ the absence of selectivity for the different \\ elements tested. Quantification of rare \\ earth elements in the biomass corresponded \\ to that expected by the mass balance, \\ proving the potential of macroalgae for the \\ removal and recovery of REE present in \\ wastewater, being a simple, economic, and \\ environmentally friendly process.
}

\begin{abstract}
A União Europeia classificou os elementos terras raras (REE) como matérias-primas críticas de elevado valor económico e interesse comercial, resultante da dificuldade e dos elevados custos na sua obtenção, mantendo um vasto campo de aplicações. Neste estudo avalia-se a utilização de diferentes macroalgas vivas, no intuito de remover e concentrar os REE a partir de águas onde estejam presentes. O potencial de utilização das macroalgas vivas foi avaliado através de ensaios cinéticos em soluções mono e multicontaminadas, incorporando os REE cério (Ce), európio (Eu), ítrio (Y), lantânio (La) e neodímio (Nd) em água salina, partindo de uma concentração inicial de $500 \mu \mathrm{g} \mathrm{L}^{-1}$, e às quais foram adicionadas as macroalgas vivas Gracilaria gracilis, Fucus vesiculosus e Ulva lactuca, em quantidades aproximadamente de 450-500 mg L-1 em peso seco. Destacaram-se as taxas de remoção alcançadas pela alga vermelha G. gracilis, com valores entre $60 \mathrm{e}$ 99\% para todos os REE em solução. As soluções multicontaminadas revelaram eficiências de remoção superiores às obtidas com as monocontaminadas, comprovando-se ainda a baixa seletividade para os diferentes elementos testados. A quantificação dos REE na biomassa está concordante com o declínio observado na solução, comprovando-se o potencial do uso de macroalgas para a remoção e recuperação dos REE presentes nas águas residuais, sendo o processo simples, económico e de baixo impacto ambiental.
\end{abstract}

\section{Introdução}

Os elementos terras raras (REE, do inglês Rare-earth elements) são constituídos pelos quinze lantanídeos: La (lantânio), Ce (cério), Pr (praseodímio), Nd (neodímio), Pm (promécio), Sm (samário), Eu (európio), Gd (gadolí- nio), Tb (térbio), Dy (disprósio), Ho (hólmio), Er (érbio), Tm (túlio), Yb (itérbio) e Lu (lutécio), a que se juntam os elementos Sc (escândio) e Y (ítrio) por apresentarem propriedades químicas semelhantes e sendo também 
encontrados nos mesmos depósitos geológicos [1].

As quantidades existentes na crosta terrestre (130 milhões de toneladas, segundo a estimativa da US Geological Service, em 2016) parecem ser insuficientes face ao incremento na utilização destes elementos nos produtos de elevada tecnologia, nomeadamente para produção de energia elétrica (turbinas eólicas, células fotovoltaicas e bombas de calor), transportes (baterias para carros híbridos e elétricos), comunicações (telemóveis e fibra ótica), tecnologia (computadores, lentes de câmaras, monitores e outros equipamentos informáticos) e sistemas de iluminação (luzes fluorescentes compactas, díodos emissores de luz) [2,3]. Os documentos apresentados pela Comissão Europeia têm enquadrado sistematicamente os REE no grupo de matérias-primas críticas, isto é, apresentam elevada importância económica no seio da União Europeia e têm elevado risco no seu fornecimento [4]. Como tal, surgiu a necessidade de incrementar processos de remoção e recuperação de REE, novos e mais eficientes, tendo como objetivo uma possível reintegração na linha de produção de novos produtos. Deste modo, consegue reduzir-se a necessidade da sua exploração mineira e respetivo impacto ambiental. 0 documento Raw Material Initiative (EU, 2011) refere a importância da aposta em metodologias de recuperação e reutilização das matérias-primas, em particular os REE a partir dos resíduos de equipamentos elétricos e eletrónicos.

A mineração leva à obtenção dos elementos requeridos, assim como excedentes [5,6]. Por exemplo, a produção de REE conduz a um excesso de La e Ce, quando a maior procura é para os elementos $\mathrm{Nd}$ e Dy [7]. Uma alternativa promissora para a obtenção destes elementos é a reciclagem de desperdícios ricos em REE. Têm sido utilizados diferentes métodos para a extração dos REE de resíduos sólidos, através de processos com uso de solventes e troca iónica [8,9]. No entanto, estes processos acarretam o inconveniente do grande consumo de reagentes químicos e a produção de elevados volumes de água residual contaminada. Para além disso, existe a dificuldade da separação dos REE devido às suas propriedades químicas análogas [7]. Nos últimos anos têm sido encetadas novas abordagens para a reciclagem e recuperação de REE com base em biotecnologias assentes na biossorção, que poderá ser reforçada com a bioacumulação quando se trata de organismos vivos [10]. 0 processo assenta normalmente na adsorção dos elementos pela biomassa que apresenta propriedades relevantes para a remoção de REE. A biossorção tem vindo a revelar-se como um método eficaz na remoção de elementos tóxicos presentes em soluções, nomeadamente quando estes se encontram em baixas concentrações. Nos últimos anos têm vindo a ser testados diferentes tipos de biossorventes, nomeadamente os constituídos por biomassa de algas, fungos, bactérias, resíduos de plantas e de animais, cascas e diversos outros [8,11-13].

No caso concreto do uso de macroalgas como biomassa, a biossorção envolve a interação de elementos químicos com as paredes celulares das algas, mediante a sua constituição química. Assim, a maior ou menor especificidade de uma macroalga para determinados elementos contaminantes é definida precisamente pela presença dos grupos funcionais nas paredes celulares. Estes variam mediante o grupo da macroalga, definindo assim, quimicamente, a sua seletividade [14]. De referir que a maioria dos estudos realizados recorrem ao uso de biomassa inerte, havendo muito poucos a explorar o potencial da biomassa viva e a possibilidade inerente da bioacumulação $[14,15]$.

0 presente estudo tem por objetivo avaliar as potencialidades das macroalgas vivas para a remoção de elementos terras raras de soluções contaminadas. Foram selecionadas as espécies Fucus vesiculosus, Gracilaria gracilis e Ulva lactuca pelas diferentes morfologias e composição das paredes celulares. Os elementos escolhidos para este estudo em soluções mono e multicontaminadas foram o Ce, o Eu, o Y, o La e o Nd.

\section{Materiais e métodos 2.1. Reagentes e soluções}

0 material de laboratório usado neste trabalho foi lavado com água da torneira e enxaguado abundantemente com água destilada, colocado em $\mathrm{HNO}_{3}$ (25\% v/v) durante 24 horas, e novamente lavado com água destilada. Utilizaram-se reagentes de qualidade analítica, sem purificações adicionais. As várias soluções dos REE foram preparadas a partir de soluções padrão comerciais da Alfa Aesar (Specpure ${ }^{\circledR}$, plasma standard solutions), nas concentrações de $1000 \pm 1 \mathrm{mg} \mathrm{L}^{-1}$ (casos dos elementos La, Y e Eu em $\mathrm{HNO}_{3}$ 5\%). As soluções de Nd foram preparadas a partir da solução comercial da Inorganic Ventures ${ }^{T M}$ de $998 \pm 2 \mathrm{mg} \mathrm{L}^{-1}$ em $\mathrm{HNO}_{3}$ 1,4\%. Também as soluções contendo o elemento Ce foram preparadas a partir da solução comercial desta marca, de $1001 \pm 7 \mathrm{mg} \mathrm{L}^{-1}$ em $\mathrm{HNO}_{3}$ 7\%.

\subsection{Biomassa}

As macroalgas utilizadas neste trabalho foram a Gracilaria gracilis, a Fucus vesiculosus e a Ulva lactuca (Figura 1). Estas algas pertencem, respetivamente, aos grupos Phaeophyta, Rhodophyta e Chlorophyta, e a sua escolha teve por base as diferenças na composição das paredes celulares, nos pigmentos presentes (que thes conferem as cores distintivas) e nos locais de acumulação dos produtos do seu metabolismo, mas também por serem um recurso abundante e de fácil acesso. Todas elas contêm clorofila e celulose na composição 
das suas paredes celulares; no entanto, diferem pela presença de polissacarídeos sulfatados, nas macroalgas vermelhas (G. gracilis); de polissacarídeos sulfatados e alginato, nas macroalgas castanhas (F. vesiculosus); e de polímeros compostos por xilose e manose, no caso das macroalgas verdes (U. lactuca) [14]. Foram recolhidas na Ria de Aveiro (40³2'57" N, $8^{\circ} 46^{\prime} 30^{\prime \prime}$ W), em período de maré baixa. Após recolha, as amostras foram transportadas para o laboratório em sacos isotérmicos contendo água do local. No laboratório foram também lavadas com água do mar filtrada para a remoção de partículas aderentes provenientes do local da colheita e mantidas em aquários arejados, em água do mar filtrada com salinidade 15, renovada e com luz natural (aproximadamente 12 horas/dia), a temperatura controlada $\left(20 \pm 2{ }^{\circ} \mathrm{C}\right)$. A água do mar usada para a manutenção das algas e para os ensaios de remoção foi recolhida no mesmo local da colheita das algas, filtrada através de filtros Millipore $^{\mathrm{TM}}$ de tamanho de poro 0,45 $\mu$ m e armazenada na ausência de luz solar direta.

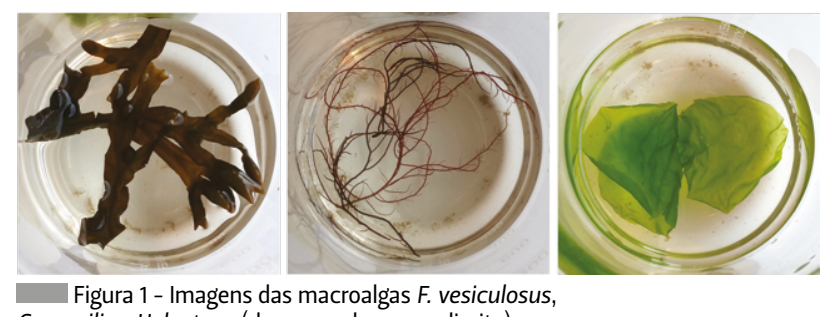

G. gracilis e U. lactuca (da esquerda para a direita).

\subsection{Desenho experimental}

\subsubsection{Ensaios comparativos de remoção}

Como ensaio inicial, foi avaliada a capacidade das três espécies de macroalgas de remover Ce, Eu, Y, La e Nd de soluções contaminadas. Para tal, colocaram-se 250 mg (peso fresco) da biomassa de F. vesiculosus, G. gracilis e U. lactuca em recipientes distintos contendo 0,1 L de uma solução contaminada com cada um dos elementos em estudo. Cada solução foi preparada com água salina de salinidade 15, obtida por diluição da água do mar filtrada com água ultrapura $(18,2 \mathrm{M} \Omega \mathrm{cm})$, à qual se adicionou o volume de uma solução concentrada por forma a obter concentrações iniciais de $500 \mu \mathrm{g} \mathrm{L}^{-1}$ de cada elemento. Posteriormente, ajustou-se ainda o pH das soluções para 7,8 por adição de solução de $\mathrm{NaOH}$ $1 \mathrm{~mol} \mathrm{~L}^{-1}$. Apesar dos efluentes com REE apresentarem concentrações muito variáveis consoante as indústrias, optou-se por considerar $500 \mu \mathrm{g} \mathrm{L}^{-1}$ como um valor indicativo de uma situação hipotética.

Após um período de estabilização de 12 horas para equilíbrio das soluções, adicionou-se cada uma das macroalgas vivas. Recolheram-se amostras da solução (alíquotas de $4 \mathrm{~mL}$ ) no momento imediatamente antes à sua adição $\left(t_{0}\right)$, e após $24\left(t_{24}\right)$ e 48 horas $\left(t_{48}\right)$. Todos os ensaios foram realizados em triplicado. As condições experimentais de temperatura e exposição à luz solar foram idênticas àquelas descritas no ponto 2.2. Os recipientes foram tapados com película Parafilm ${ }^{\circledR}$ durante o período experimental para reduzir perdas por evapotranspiração e não foi utilizada agitação. Em paralelo com estas experiências, foram realizados, nas mesmas condições de exposição solar e temperatura, ensaios de controlo (soluções contaminadas com cada um dos REE e ausência de macroalga) e de brancos (macroalga em água salina sem adição de REE). As amostras das diversas experiências foram acidificadas a $\mathrm{pH} \leq 2$ com solução $\mathrm{HNO}_{3}(65 \%)$ para posterior quantificação dos REE.

\subsubsection{Ensaios cinéticos com Gracilaria gracilis} Realizaram-se ensaios cinéticos apenas com a macroalga G. gracilis por ser a mais eficiente na remoção de REE. Manteve-se a mesma proporção entre a biomassa de macroalgá por volume de solução $\left(2500 \mathrm{mg} \mathrm{L}^{-1}\right)$ e a concentração inicial de $500 \mu \mathrm{g} \mathrm{L}^{-1}$ para cada elemento. No entanto, a massa de alga e o volume final de solução foram aumentados para 2500 mg e $1 \mathrm{~L}$, respetivamente. Isto permite a recolha de alíquotas nos diferentes tempos sem afetar significativamente a concentração dos elementos em solução. Foram realizados dois tipos de ensaios: soluções contaminadas com cada um dos REE e soluções contaminadas com a mistura de cinco REE (Ce, Eu, Y, La e Nd) com igual concentração $\left(500 \mu g \mathrm{~L}^{-1}\right)$.

0 procedimento da exposição das macroalgas às soluções contaminadas, assim como os controlos e brancos, foi semelhante aos ensaios anteriores. As amostras de água foram recolhidas (alíquotas de $4 \mathrm{~mL}$ ) nos tempos 0, 1, 3, 6, 12, 24 e 48 horas, com monitorização da variação do pH (controlos no início, a 6 horas, após 24 horas e após 48 horas). Todos os ensaios foram realizados em triplicado. As macroalgas foram pesadas no início e no final dos ensaios para avaliação da taxa de crescimento, sendo então congeladas em $\mathrm{N}_{2}$ líquido, liofilizadas e solubilizadas através de ataque ácido para posterior quantificação dos REE presentes na sua biomassa.

\subsection{Determinações analíticas}

A caracterização da água do mar recolhida na Ria de Aveiro foi realizada com condutivímetro (WTW, modelo 330i), determinando-se a salinidade $(33,7)$ e a condutividade $\left(51,7 \mathrm{mS} \mathrm{cm}^{-1}\right)$, e depois o pH (medidor WTW inoLab pH 720), registando-se o valor 8,1.

A determinação dos REE nas águas dos ensaios foi realizada com recurso ao equipamento de espectrometria de massa acoplado com fonte de plasma induzido (ICP-MS), no Laboratório Central de Análises da Universidade de Aveiro (ICP-MS THERMO X Series, 
com nebulizador Burgener a $1 \mathrm{~mL} \mathrm{~min}^{-1}$ e cones de níquel) [16]. Foram usadas curvas de calibração obtidas a partir de soluções padrão de concentração 0,$1 ; 0,5 ; 1 ; 2 ; 5 ; 10 ; 20 ; 50$ e $100 \mu \mathrm{g}^{-1}$, preparadas por diluição do padrão comercial multielementar com $\mathrm{HNO}_{3}$ a $2 \%$ ( $\left.\mathrm{v} / \mathrm{v}\right)$. 0 erro de cada padrão não excedeu os $10 \%$ e apenas foram aceites retas de calibração com um coeficiente de correlação mínimo $\left(R^{2}\right)$ de 0,999. O limite de quantificação de cada elemento foi de $0,1 \mu \mathrm{g} \mathrm{L}^{-1}$, sendo que este valor corresponde ao padrão menos concentrado da reta de calibração.

O teor de REE nas macroalgas foi igualmente determinado por ICP-MS após a respetiva digestão ácida. Foram colocados $200 \mathrm{mg}$ da amostra num tubo de teflon $+2 \mathrm{~mL}$ de $\mathrm{HNO}_{3}(65 \% \mathrm{v} / \mathrm{v})$, realizando-se um primeiro ciclo num micro-ondas modelo CEM Mars 5 durante 15 minutos (5 minutos a temperatura crescente até $170{ }^{\circ} \mathrm{C}$, que é então mantida durante 10 minutos), após o que é arrefecido. Adicionou-se então $250 \mu \mathrm{L}$ de $\mathrm{H}_{2} \mathrm{O}_{2}(30 \% \mathrm{~m} / \mathrm{m})$, seguindo-se novo ciclo de micro-ondas. No final obteve-se uma solução aquosa que foi recolhida num tubo de polietileno de $25 \mathrm{~mL}$, cujo volume foi perfazido com água ultrapura. Foi feita também a quantificação de brancos da digestão (mesmos reagentes, sem amostra) e de material de referência certificado (IAEA 140/TM - Trace Elements and Methilmercury in Seaweed (Fucus sp.), executadas em simultâneo com as amostras. No entanto, devido à dificuldade em encontrar materiais de referência que contenham estes elementos em matrizes vegetais, apenas foi possível obter percentagens de recuperação para os elementos La e Ce $(83 \pm 1 \%$, para um conjunto de seis réplicas) no material de referência utilizado. Devido à similaridade química entre os elementos terras raras, foi assumido que a recuperação dos restantes elementos testados seria semelhante. Os dados foram corrigidos para todos os elementos tendo em conta estes valores.

As concentrações dos REE nas águas da Ria de Aveiro estavam abaixo do limite de quantificação. Os teores nas macroalgas recolhidas na Ria foram próximos ou abaixo do limite de quantificação do equipamento. Procedeu-se ainda à determinação da razão entre o peso fresco e o peso seco das macroalgas, pesando várias amostras em balança analítica (peso fresco), posteriormente secas ao ar durante alguns dias e novamente pesadas, até as mesmas registarem peso constante (peso seco).

\subsection{Fórmulas}

A taxa de crescimento relativo (TCR) da G. gracilis de REE foi calculada, na presença e na ausência de REE, através da equação 1 [17]:

$$
\operatorname{TCR}\left(\% \mathrm{dia}^{-1}\right)=\left[\ln \left(\mathrm{m}_{\mathrm{t}} / \mathrm{m}_{0}\right)\right] / \mathrm{t} \times 100
$$

O termo $m_{0}$ representa a massa inicial da macroalga usada, $m_{t}$ a massa da macroalga no final da experiência, e t a duração da experiência (dias). Deve notar-se que a eq. 1 assume um crescimento exponencial e, por essa razão, uma TCR de, por exemplo, 100 \% dia-1 não significa que a macroalga duplicou a sua biomassa, mas sim que esta aumentou aproximadamente 2,7 (número de Euler) o seu valor inicial.

Admitindo que não existem perdas do elemento, a eficiência da biorremoção pela biomassa foi avaliada através da percentagem de REE removida da solução de acordo com a equação 2:

$$
\text { \% remoção }=\left[\left(C_{0}-C_{t}\right) / C_{0}\right] \times 100
$$

$C_{0}$ representa a concentração inicial e $C_{t}$ a concentração no instante $t$ do elemento terra rara em solução.

Nestas condições, o elemento removido da solução que se encontra na biomassa $\left(q_{t}\right)$ é dada pela equação 3:

$$
q_{t}\left(\mu g^{\prime} g^{-1}\right)=\left[\left(C_{0}-C_{t}\right) \times V\right] / M
$$

$V$ indica o volume da solução (L) e $M$ a massa de alga (g) em peso seco.

A descrição da cinética de remoção de REE pela macroalga na solução foi feita recorrendo ao ajuste dos dados experimentais a três modelos cinéticos de referência, na sua forma não linear, com recurso ao software Graphpad Prism 6. Os modelos cinéticos e as respetivas equações foram os seguintes:

Pseudo-primeira ordem (Lagergren):

$q_{t}=q_{e}\left(1-e^{-k_{1} t}\right)$

Pseudo-segunda ordem ( $\mathrm{Ho})$ :

$$
q_{t}=\frac{q_{e}^{2} K_{2} t}{1+q_{e} K_{2} t}
$$

Elovich:

$$
q_{t}=\frac{1}{\beta} \ln (1+\alpha \beta t)
$$

O termo $q_{t}$ representa a quantidade de REE sorvido por grama de macroalga no tempo $t\left(\mu g g^{-1}\right), q_{e} a$ quantidade de REE sorvido por grama de macroalga no equilíbrio $\left(\mu g^{\prime}{ }^{-1}\right), k_{1}$ a constante de velocidade $d a$ cinética de pseudo-primeira ordem $\left(h^{-1}\right), k_{2}$ a constante de velocidade da cinética de pseudo-segunda ordem $\left(g \mu g^{-1} h^{-1}\right), \alpha$ a velocidade inicial de sorção $\left(\mu g g^{-1} h^{-1}\right)$, e $\beta$ a constante de dessorção $\left(g \mu g^{-1}\right)$. 

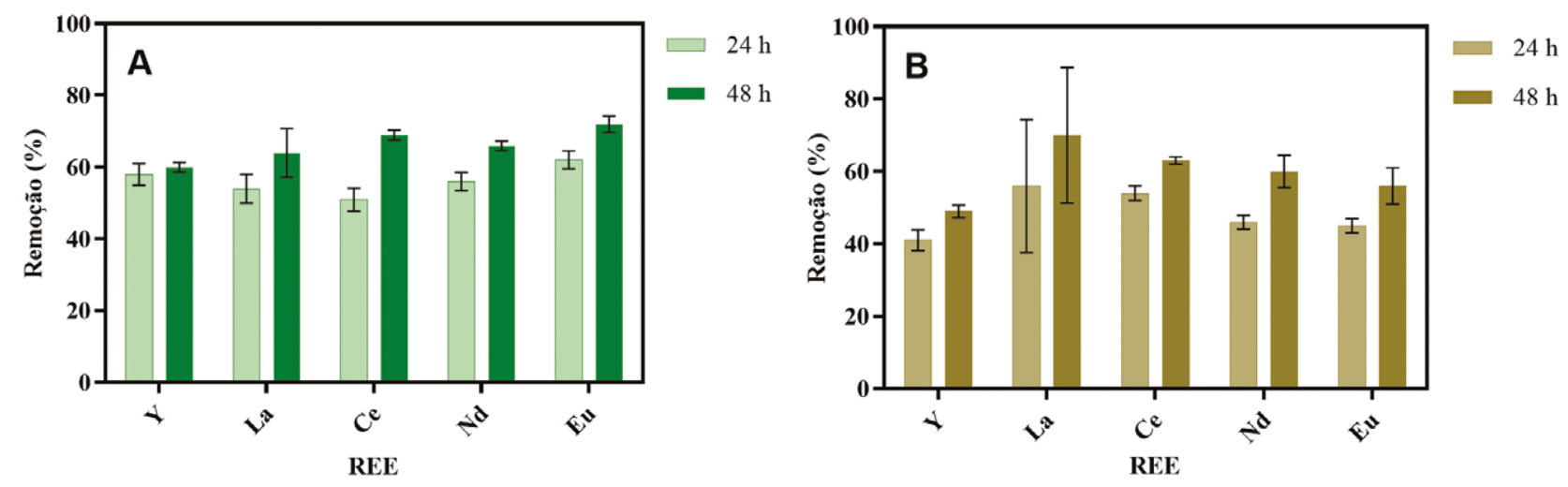

Figura 2 - Percentagens médias de remoção de REE obtidas para as macroalgas U. lactuca (A), F. vesiculosus (B) e G. gracilis (C) após 24 e 48 horas de contacto com soluções monocontaminadas por REE. As barras de erro correspondem ao desvio padrão $(n=3)$. Concentração inicial de cada REE $=500 \mu \mathrm{g} \mathrm{L}^{-1}$; quantidade de alga $=2500 \mathrm{mg} \mathrm{L}^{-1}$ (peso fresco); salinidade $=15 \mathrm{e} \mathrm{pH}$ inicial $=7,8$.

\section{Resultados e discussão}

\subsection{Redução da concentração de terras raras em solução na presença de macroalgas}

A Figura 2 mostra as percentagens médias de remoção dos elementos Y, La, Ce, Nd e Eu de soluções monocontaminadas na presença de U. lactuca, $G$. gracilis e $F$. vesiculosus, após 24 horas e 48 horas. Dado que as concentrações dos REE nas experiências de controlo (sem macroalgas) não apresentaram variações consideráveis ao fim de 24 ou 48 horas (< $7 \%$ da concentração inicial), assumiu-se que os decréscimos observados na solução resultaram da remoção pelas macroalgas. As percentagens médias de remoção pela U. lactuca dos elementos em solução foram relativamente uniformes, $50-60 \%$ ao fim de 24 horas ou $60-70 \%$ em 48 horas. Nos casos da G. gracilis e $F$. vesiculosus, os intervalos ao fim de 48 horas de exposição foram ligeiramente maiores, $45-70 \%$ e $60-80 \%$, respetivamente. Os incrementos de remoção
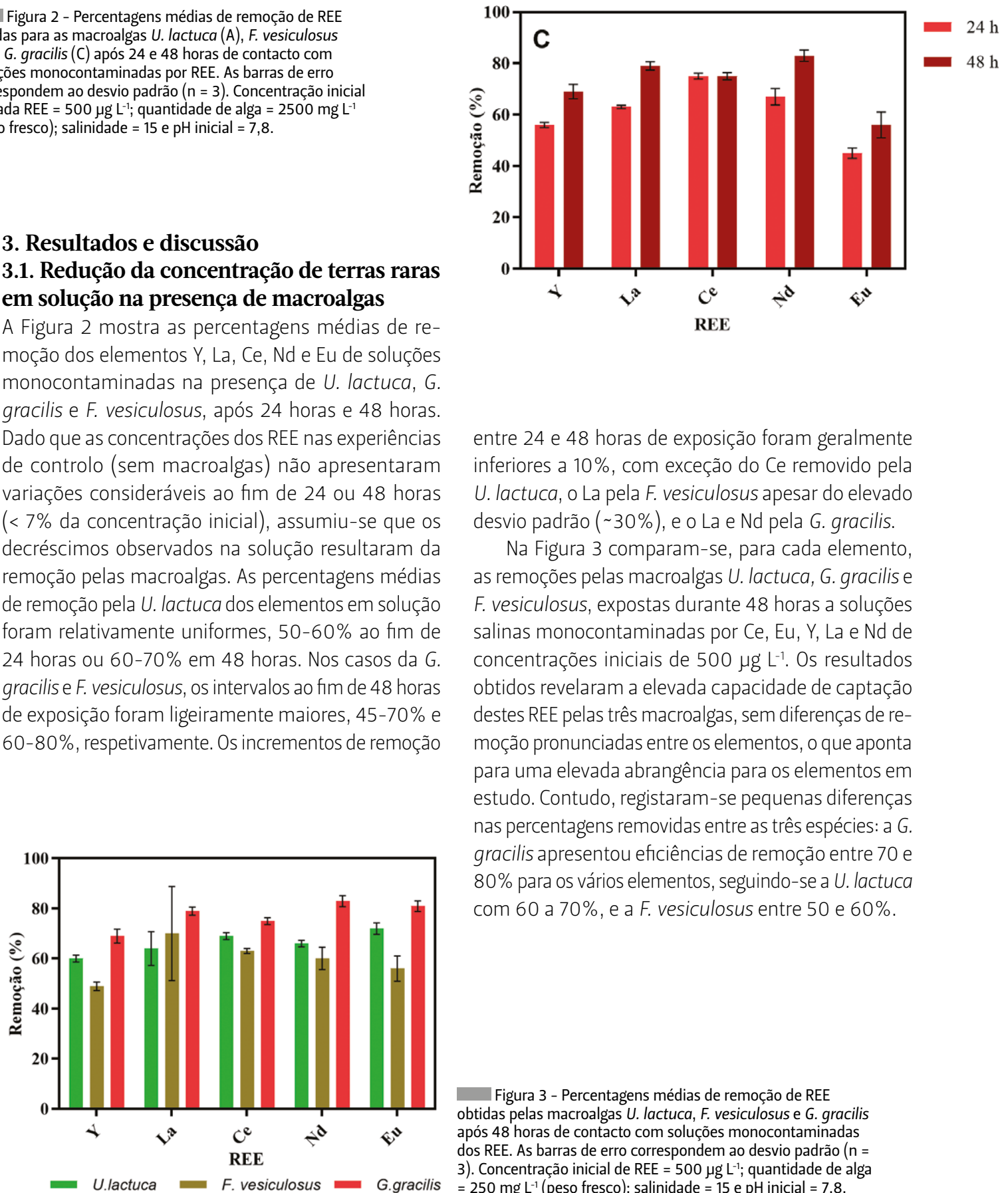

entre 24 e 48 horas de exposição foram geralmente inferiores a 10\%, com exceção do Ce removido pela U. lactuca, o La pela F. vesiculosus apesar do elevado desvio padrão ( 30\%), e o La e Nd pela G. gracilis.

$\mathrm{Na}$ Figura 3 comparam-se, para cada elemento, as remoções pelas macroalgas U. lactuca, G. gracilise F. vesiculosus, expostas durante 48 horas a soluções salinas monocontaminadas por Ce, Eu, Y, La e Nd de concentrações iniciais de $500 \mu \mathrm{g} \mathrm{L} \mathrm{L}^{-1}$. Os resultados obtidos revelaram a elevada capacidade de captação destes REE pelas três macroalgas, sem diferenças de remoção pronunciadas entre os elementos, o que aponta para uma elevada abrangência para os elementos em estudo. Contudo, registaram-se pequenas diferenças nas percentagens removidas entre as três espécies: a $G$. gracilis apresentou eficiências de remoção entre 70 e $80 \%$ para os vários elementos, seguindo-se a U. lactuca com 60 a $70 \%$, e a F. vesiculosus entre 50 e $60 \%$.

Figura 3 - Percentagens médias de remoção de REE obtidas pelas macroalgas U. lactuca, F. vesiculosus e $G$. gracilis após 48 horas de contacto com soluções monocontaminadas dos REE. As barras de erro correspondem ao desvio padrão $(n=$ 3). Concentração inicial de REE $=500 \mu \mathrm{g} \mathrm{L}^{-1}$; quantidade de alga 


\subsection{Características das macroalgas}

As diferentes remoções dos elementos em solução pelas macroalgas poderão resultar das desigualdades da área superficial, da estrutura de cada espécie e da presença dos diferentes grupos funcionais nas suas superfícies, capazes de condicionar a afinidade para os elementos presentes. De entre as macroalgas testadas, em comum verifica-se a presença de clorofila e celulose na composição das suas paredes celulares. No entanto, a área superficial da U. lactuca ( $264 \mathrm{~cm}^{2} \mathrm{~g}^{-1}$ ) excede largamente a registada para a $\mathrm{G}$. gracilis $\left(79 \mathrm{~cm}^{2} \mathrm{~g}^{-1}\right)$ e F. vesiculosus $\left(30 \mathrm{~cm}^{2} \mathrm{~g}^{-1}\right)$, como registado em trabalho anterior [18]. Verificam-se ainda outras diferenças, como a presença de polissacarídeos sulfatados e alginato nas macroalgas castanhas ( $F$. vesiculosus), de polissacarídeos sulfatados, xilanos e galactanos nas macroalgas vermelhas (G. gracilis) e de polímeros compostos por xilose e manose, no caso das macroalgas verdes (U. lactuca) $[14,18]$.

As diferenças estruturais, físicas e químicas, das macroalgas testadas podem explicar as diferentes remoções obtidas dos elementos em solução. Por exemplo, sendo a área exterior de contacto da $U$. lactuca muito maior que a registada para a G. gracilis e da própria F. vesiculosus, tal pressuporia uma maior disponibilidade para a remoção dos elementos terras raras pela primeira. No entanto, neste trabalho observou-se ter sido a Gracilaria gracilis a mais eficaz. Tal poderá dever-se a uma maior dispersão desta macroalga por toda a solução, com os seus filamentos ramificados a permitirem um maior preenchimento de todo o volume da solução, "compensando" desse modo a menor área de contacto disponível com uma melhor distribuição por toda a solução. Presume-se também que a presença de grupos funcionais com grande afinidade para os REE poderá ter maior relevância na sua remoção pela G. gracilis, bem como uma possível maior seletividade para os REE analisados (Figura 2). (Para informação mais detalhada sobre os grupos funcionais presentes nestas espécies consultar $[18,19])$.

\subsection{Variação da biomassa de Gracilaria} gracilis e do $\mathbf{p H}$ da solução durante os ensaios Atendendo aos resultados obtidos ao fim de $24 \mathrm{e}$ 48 horas com as três macroalgas, optou-se pela G. gracilis para a continuidade dos ensaios. Assim, foram efetuadas várias pesagens para a obtenção da razão entre o peso fresco e o peso seco desta macroalga, obtendo-se o valor médio de $0,18 \pm 0,024(n=10)$ que está em concordância com o reportado em outros estudos (por exemplo, [18]). Determinou-se a sua taxa de crescimento através da equação 1, com o objetivo de avaliar as alterações de biomassa durante os ensaios e a influência da exposição aos REE. As taxas de crescimento relativo (TCR, \% $\mathrm{dia}^{-1}$ ) medidas nos brancos (macroalga em água salina sem adição de REE) e nas diferentes soluções contaminadas foram as seguintes: nos brancos entre 6,0 e $6,5 \% \mathrm{dia}^{-1}$, nas soluções monocontaminadas entre 6,0 e 6,8\% dia $^{-1}$ e na solução multicontaminada $8,6 \% \mathrm{dia}^{-1}$. De referir que não foram adicionados nutrientes ao longo do tempo. Os valores obtidos são próximos dos reportados por Pickering [20]. A ausência de diferenças significativas entre as TCR dos brancos e das soluções com REE (unpaired t test, two tailed $p<0,05$ ) sugere que a presença dos REE não influenciou o normal desenvolvimento da macroalga durante os ensaios.

$\mathrm{O}$ pH das soluções salinas contaminadas durante o decorrer da exposição da macroalga G. gracilis a soluções mono e multicontaminadas revelou pequenos incrementos entre $\mathrm{t}=0 \mathrm{e} \mathrm{t}=24$ horas, com valores médios de 8,7, que se manteve para $\mathrm{t}=48$ horas. Nos controlos, o pH manteve-se constante, ligeiramente abaixo de 8,0. Este aumento inicial pode ser devido ao metabolismo da macroalga, com a fixação e consumo de $\mathrm{CO}_{2}$ a partir do ião hidrogenocarbonato $\left(\mathrm{HCO}_{3}{ }^{-}\right)$no seu processo fotossintético, que supera a libertação durante o processo de respiração noturna das macroalgas [21].

\subsection{Estudos cinéticos de remoção com a macroalga Gracilaria gracilis}

Procedeu-se à realização dos ensaios cinéticos da $G$. gracilis com os elementos Ce, Eu, Y, La e Nd. Foram realizados estudos comparativos em soluções contaminadas com um único elemento (Ce, Eu, Y, La ou Nd) e com o conjunto destes elementos. As concentrações iniciais de cada elemento foram semelhantes, $500 \mu \mathrm{g} \mathrm{L}^{-1}$, e a proporção entre a massa de macroalga e o volume da solução foi a mesma $\left(2,5 \mathrm{~g} \mathrm{~L}^{-1}\right)$.

As razões entre a concentração do elemento no tempo $t\left(C_{t}\right)$ e a concentração inicial $\left(C_{0}\right)$ para os diversos tempos de exposição da macroalga apresentam-se na Figura 4. Para cada tempo t comparam-se as razões em soluções mono e multicontaminadas. 0 declínio da razão $C_{t} / C_{0}$ aponta para uma curva do tipo hipérbole negativa, com os maiores decréscimos a ocorrerem nas primeiras seis horas de exposição da macroalga às soluções contaminadas. A maior velocidade de remoção inicial poderá dever-se ao facto da macroalga apresentar a sua superfície livre dos elementos terras raras, havendo, por isso, um elevado gradiente de concentrações entre a macroalga e a solução. A difusão dos catiões na direção da superfície facilita a captação passiva, de natureza físico-química. À medida que decorre a adsorção à superfície ou incorporação dos elementos, o seu gradiente diminui e, portanto, também o fluxo difusivo. 0 presente estudo não permite elucidar se, numa fase subsequente, haverá a transferência de algum ou alguns REE para 
o interior das células através do metabolismo da alga.

Comparando os comportamentos obtidos para cada REE nas soluções mono e multicontaminadas, verifica-se que a presença dos outros REE na solução não afeta a cinética de remoção do Y e Ce (unpaired $t$ test, two-tailed $p<0,05)$. No entanto, para o Eu, o Nd e o La, observou-se uma remoção ligeiramente mais rápida quando estavam mais elementos em solução. Também não foi possível elucidar as causas para esta aparente sinergia.

Os ensaios de controlo (soluções contaminadas com cada um dos REE, sem a presença da macroalga) confirmaram que as perdas por outros motivos, como sejam a adsorção nos frascos de vidro ou formação de precipitados e coloides foram praticamente inexistentes (Figura 4). Foram exceções o Ce (nas soluções mono e multicontaminadas), que revelou uma diminuição de $7 \%$ na razão $C_{t} / C_{0}$, assim como o $Y$ e o Eu nas soluções multicontaminadas ( 9 e 6\%, respetivamente). Também a presença de REE nos brancos (soluções com a presença das macroalgas, sem adição de REE) estiveram sempre abaixo de $1 \mu \mathrm{g}^{\mathrm{L}} \mathrm{L}^{-1}$, confirmando a inexistência de contaminações.

\subsection{Aplicação de modelos cinéticos}

Com base no declínio das concentrações em solução monocontaminadas, foram estimadas as quantidades de cada elemento incorporado pela macroalga $G$. gracilis, $q_{t}$ para os diversos tempos $t$, através da equação 3. Os va-
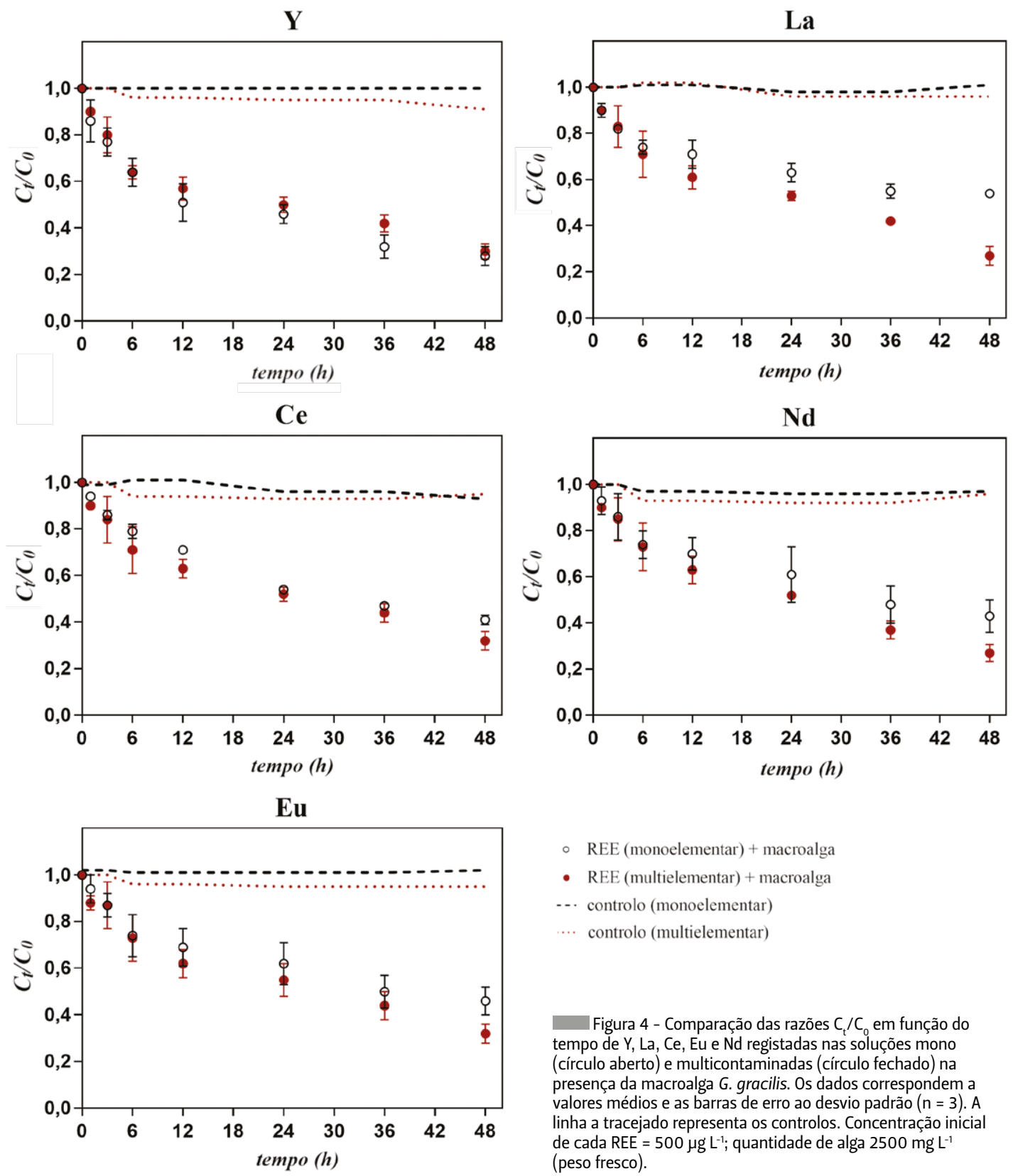

\footnotetext{
- REE (monoelementar) + macroalga

- REE (multielementar) + macroalga

-.. controlo (monoelementar)

‥ controlo (multielementar)
}

Figura 4 - Comparação das razões $C_{t} / C_{0}$ em função do
tempo de $Y$, La, Ce, Eu e Nd registadas nas soluções mono
(círculo aberto) e multicontaminadas (círculo fechado) na
presença da macroalga $G$. gracilis. Os dados correspondem a
valores médios e as barras de erro ao desvio padrão $(n=3)$. A
linha a tracejado representa os controlos. Concentração inicial
de cada $\mathrm{REE}=500 \mu \mathrm{g} \mathrm{L}^{-1}$; quantidade de alga $2500 \mathrm{mg} \mathrm{L}^{-1}$
(peso fresco). 
$\mathbf{Y}$

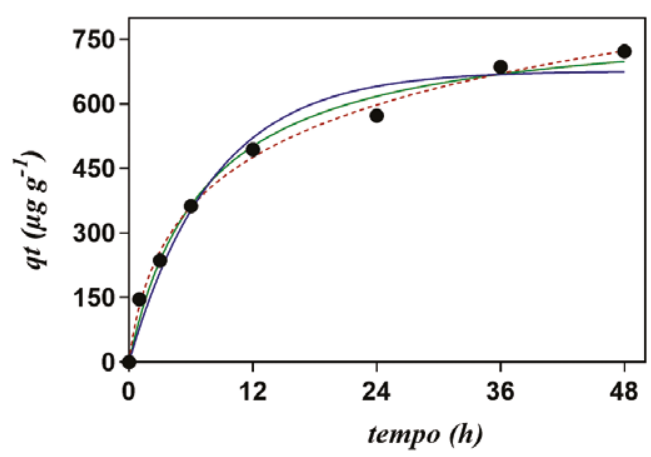

Ce

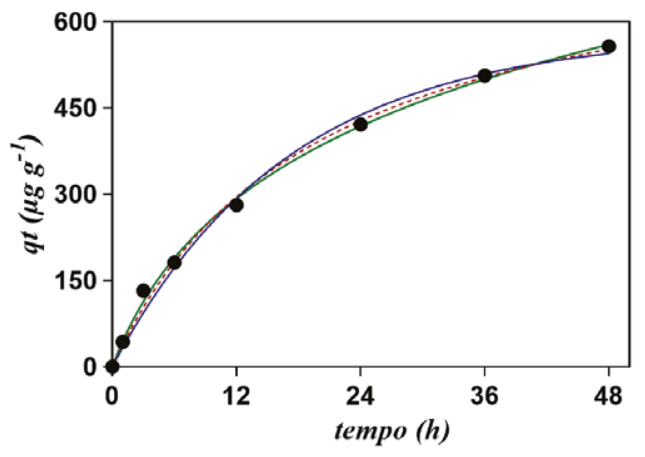

lores obtidos são apresentados na Figura 5. Procedeu-se à modelação cinética da retenção de REE com recurso ao ajuste dos dados obtidos com os modelos PFO, PSO e Elovich (Figura 5). O modelo de Elovich foi o que melhor se ajustou aos dados obtidos experimentalmente $\left(R_{2}\right.$ mais elevado - 0,9934-0,9981 - e valores de $S_{y . x}$ mais baixos - 9,509-18,20). Estes resultados sugerem haver mais do que um mecanismo de natureza química na sorção dos REE (quimiossorção), baseados na interação entre os REE e os grupos funcionais presentes à superfície da macroalga [14]. Foi igualmente feito o ajuste dos modelos cinéticos aos dados obtidos para as soluções multicontaminadas (não representado), bem como os valores dos parâmetros cinéticos. Mais uma vez foi o modelo cinético de Elovich que melhor se ajustou aos dados experimentais, confirmado pelos valores de $\mathrm{R}_{2}$ mais elevados $(0,9887-0,9921)$ e os valores de $S_{y . x}$ mais reduzidos $(23,32-30,15)$. 0 modelo cinético de PFO foi o que teve piores ajustes $\left(0,9616<R_{2}<0,9711\right.$ e $42,84<S_{y . x}<52,43$ ).

\subsection{Comparação entre a quantidade de REE presentes na biomassa e a quantidade estimada}

Ao fim de 48 horas, verificou-se que as percentagens de remoção para cada um dos REE não apresentaram diferenças assinaláveis no caso da solução multicontaminada ( 70\%), enquanto nas soluções monocontaminadas a remoção variou entre $46 \%$ para o La e $70 \%$ para o Y (Figura 6).
La
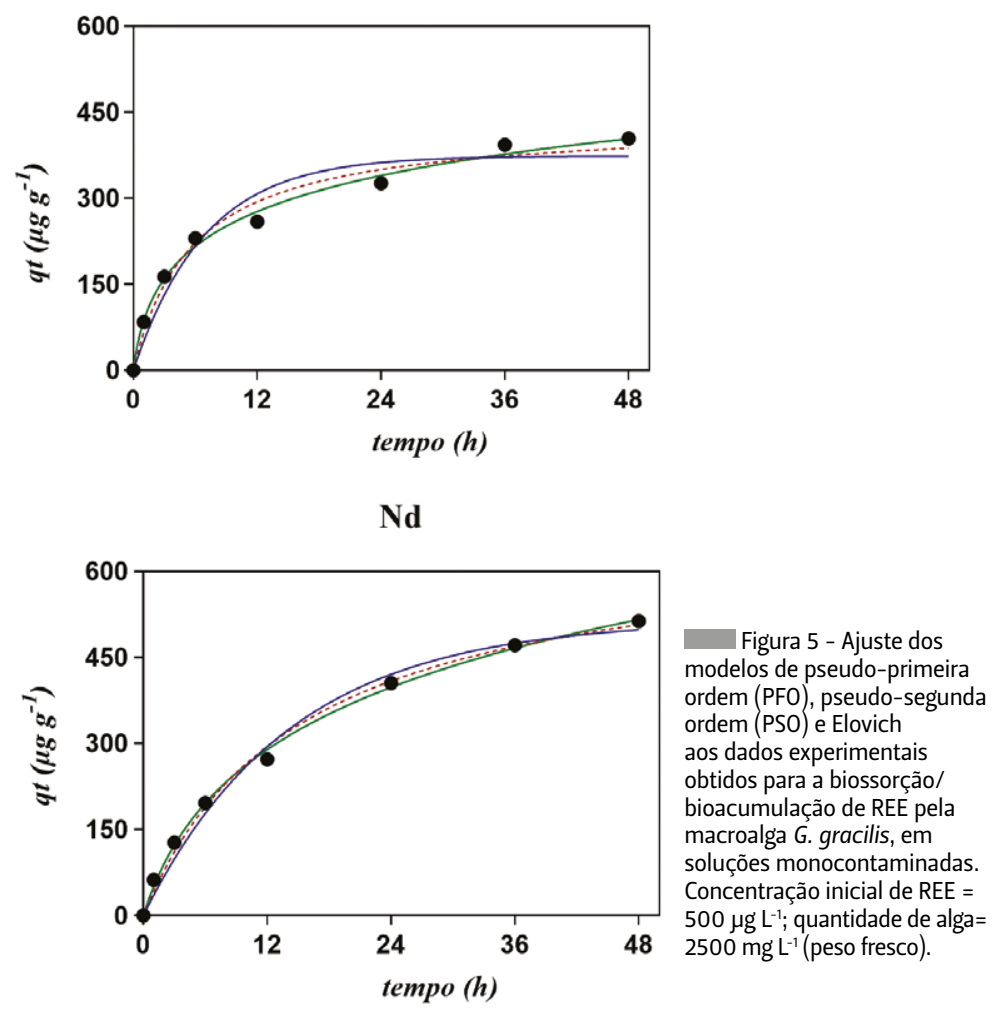

Eu

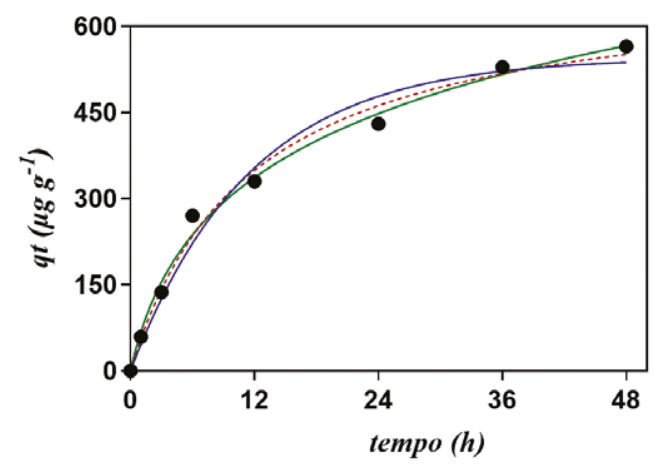

- PFO

-.... PSO

- Elovich

Figura 6 - Comparação das eficiências de remoção de Y, La, Ce, Nd e Eu pela G. gracilis ao fim de 48 horas em solução mono e multicontaminada.

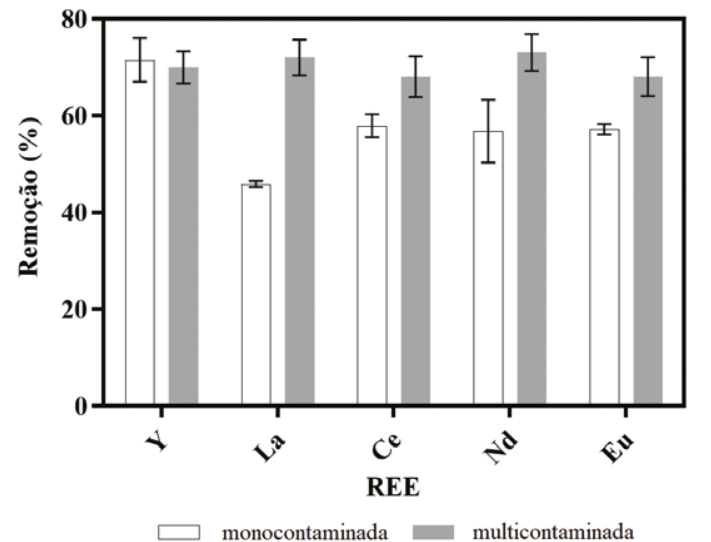

QUÍMICA | Vol. 45 | N. ${ }^{0} 163$ | 2021 | 289 
A quantificação dos REE na biomassa das três macroalgas mostrou que as concentrações presentes, após 48 horas, foram duas a três ordens de grandeza superiores às concentrações iniciais, apontando para a capacidade das macroalgas (e em particular a selecionada, G. gracilis) na remoção de REE existentes em águas contaminadas, bem como da sua concentração numa pequena quantidade de biomassa. Os dados obtidos foram expressos nos gráficos da Figura 7, que comparam a concentração dos elementos na biomassa de G. gracilis obtida pela quantificação na biomassa ( $q_{f}$ obtido) com os valores obtidos através da equação 3 ( $q_{f}$ estimado), em ambas as condições (soluções monocontaminadas e soluções multicontaminadas).

Nas soluções monocontaminadas, os conteúdos dos vários REE detetados e esperados na biomassa foram, respetivamente: $Y=748 \pm 69$ e $722 \pm 87 \mu g^{\prime-1}$; $\mathrm{Ce}=549 \pm 28$ e $557 \pm 32 \mu \mathrm{g} \mathrm{g}^{-1} ; \mathrm{Nd}=496 \pm 33$ e $513 \pm 84 \mu \mathrm{g}^{\prime} \mathrm{g}^{-1}$; e $E u=589 \pm 18$ e $565 \pm 33 \mu \mathrm{g} \mathrm{g}^{-1}$. Estes não diferem estatisticamente $(p>0,05)$. Já no caso do La, o valor medido $\left(490 \pm 47 \mu \mathrm{g}^{\mathrm{g}} \mathrm{g}^{-1}\right)$ foi significativamente superior ao esperado $\left(404 \pm 8 \mu \mathrm{gg}^{-1}\right.$, com o valor $p=$ $0,0354<0,05)$. Nas soluções multicontaminadas, nenhum REE diferiu significativamente $(p<0,05)$ entre o valor medido e estimado.

Estes resultados confirmam que praticamente a totalidade dos REE removidos da solução foram incorporados pela macroalga, registando-se uma maior discrepância (entre o $q_{f}$ estimado e o $q_{f}$ obtido) para com o La (erro relativo de $17 \%$ ) e para o Eu (erro relativo de $6 \%$ ), com a alga a apresentar níveis inferiores aos previstos pelo balanço de massa. 0 maior teor obtido foi com o $Y\left(q_{f}\right.$ obtido $=722 \pm 88$ $\left.\mu g g^{-1}, n=3\right)$ e o menor foi com o La ( $q_{f}$ obtido $=404 \pm 8$ $\left.\mu g g^{-1}, n=3\right)$, ambos nas soluções monocontaminadas.

Figura 7 - Concentração final de Y, La, Ce, Nd e Eu na biomassa da macroalga $G$. gracilis (valor médio), após exposição a soluções monocontaminadas (a) e multicontaminadas

(b), estimadas através da equação (3) e obtidas através de quantificação na macroalga. As barras de erro representam o desvio padrão ( $n=3, q_{\mathrm{f}}$ estimado; $n=6, q_{\mathrm{f}}$ obtido).



\subsection{Competição ou sinergia entre REE na sorção à Gracilaria gracilis?}

A adsorção de um elemento ou composto a uma superfície depende, para além das propriedades intrínsecas da superfície do sorvente e das condições do meio, de um balanço entre a disponibilidade do elemento/ composto em solução para migrar para a superfície do sobrante e dos locais onde a adsorção tem lugar.

Os resultados obtidos mostram essa capacidade que a macroalga G. gracilis tem em remover vários REE de soluções, concentrando-os nos seus tecidos. Também mostram que, na presença de cinco REE em solução (concentração total de $5 \times 500 \mu \mathrm{g}^{\mathrm{L}} \mathrm{L}^{-1}$ ), a quantidade adsorvida de cada elemento supera a quantidade de quando apenas um REE está presente, embora com ligeiras diferenças entre os elementos. Estas constatações sugerem que os locais de adsorção da macroalga presentes na solução não são limitantes, mesmo quando se passa de $500 \mu \mathrm{g}^{\prime-1}$ por elemento para os $2500 \mu \mathrm{g} \mathrm{L}^{-1}$ (vários elementos, contabilizando todos os presentes na solução multicontaminada). Também mostram que não terá existido evidência de competição entre os elementos para se fixarem nos locais de adsorção.

A não preferência da macroalgá por alguns dos REE testados (competição) é percetível, se atendermos ao facto de estes não serem significativamente diferentes entre si (em termos químicos), e ainda menos quando presentes no seu estado hidratado. Também é indicador da disponibilidade que a macroalga apresenta para a captação dos REE, adsorvendo-os e posteriormente incorporando-os no seu interior (processo de bioacumulação), libertando assim os grupos ativos presentes nas membranas para novas lig̉ações. Como tal, o recurso às macroalgas marinhas para remoção de REE presentes em soluções multicontaminadas apresenta-se como uma alternativa eficaz para regenerar essas águas poluídas (e eventualmente recuperar esses REE).

Com base neste trabalho, o grupo de investigação da UA tem vindo a desenvolver vários outros estudos

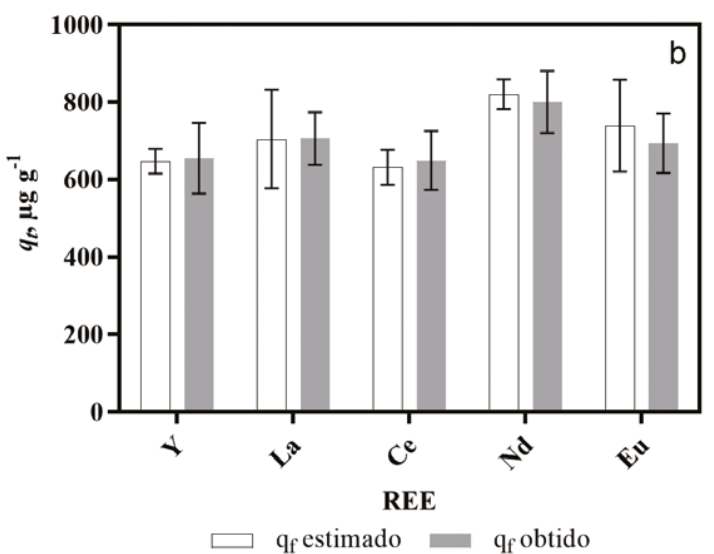


dando ênfase ao uso das macroalgas, confirmando a sua capacidade e apetência para a remoção dos poluentes tóxicos convencionais, como o $\mathrm{Cd}$, $\mathrm{Cr}, \mathrm{Cu}, \mathrm{Hg}$, Ni e P $[22,23]$, mas igualmente para a remoção de REE $[24,25]$.

\section{Conclusões}

Os resultados destacam o potencial do uso da macroalga viva G. gracilis na remoção de REE das águas residuais mesmo quando em misturas mais ou menos complexas e de baixa concentração, apontando para o efetivo sucesso desta solução face a uma das maiores dificuldades encontradas na recuperação e reutilização destes elementos. Conseguiram-se eficiências de remoção de até $70 \%$ em 48 horas, e foram recuperadas praticamente $100 \%$ de todos os elementos presentes ( $\mathrm{Y}, \mathrm{Ce}, \mathrm{Nd}$, Eu e La) na biomassa da alga, numa solução 280 vezes mais concentrada (soluções monocontaminadas) e até 400 vezes mais concentrada (soluções multicontaminadas).

Embora a G. gracilis não tenha mostrado grande seletividade para qualquer dos elementos em estudo, as elevadas eficiências de remoção realizadas nas soluções multicontaminadas e a sua capacidade de sobrevivência à exposição dos elementos, tornam estas macroalgas agentes muito promissores para a sua recuperação. Mais concretamente, a G. gracilis aqui estudada poderá ter um enorme potencial para a biorremediação e recuperação de REE em efluentes com baixas concentrações, reduzindo o seu impacto poluidor no meio ambiente e, simultaneamente, minorar a dependência da mineração, com todos os problemas ambientais associados e elevados custos de exploração, para se obterem estes elementos críticos. Poderá, inclusivamente, reduzir a dependência dos restantes países à hegemonia da China.

\section{Agradecimentos}

Este trabalho foi financiado pela Fundação para a Ciência e a Tecnologia (FCT), I.P. e Ministério da Educação (MEC) através de fundos nacionais, e cofinanciado pelo FEDER, no âmbito do Acordo de Parceria PT2020 e Compete 2020, pelo apoio financeiro ao REQUIMTE, CIIMAR e CESAM (UIDB/50006/2020, UID/Multi/04423/2019, e UIDB/50017/2020 + UIDP/50017/2020, respetivamente). Bruno Henriques agradece à $\mathrm{FCT}$ o contrato de investigação (CEECIND/03511/2018) no âmbito do CEEC Individual 2018, enquanto João Pinto e Jéssica Jacinto agradecem as suas bolsas de doutoramento (2020.05323.BD e UI/BD/151290/2021, respetivamente).

\section{Referências}

[1] V. Balaram, Geosci. Front. 2019, 10, 1285-1303. DOI: 10.1016/j. gsf.2018.12.005.

[2] M. A. Müller, D. Schweizer, V. Seiler, J. Bus. Ethics 2016, 138, 627-648. DOI: 10.1007/s10551-015-2773-3.

[3] F. Zhao, E. Repo, Y. Meng, X. Wang, D. Yin, M. Sillanpää, J. Colloid Interface SCi. 2016, 465, 215-224. DOI: 10.1016/j.jcis.2015.11.069.

[4] G. A. Blengini, C. E. L. Latunussa, U. Eynard, C. Torres de Matos, D. Wittmer, K. Georgitzikis, C. Pavel, S. Carrara, L. Mancini, M. Unguru, D, Blagoeva, F. Mathieux, D. Pennington, Study on the EU's List of Critical Raw Materials (2020): Final Report; European Commission (Ed.); European Union: Brussels, 2020. DOI: $10.2873 / 904613$.

[5] R. Schulze, B. P. Weidema, L. Schebek, M. Buchert, Resour. Conserv. Recycl. 2018, 134, 336-346. DOI: 10.1016/j.resconrec.2017.11.006.

[6] K. Binnemans, P. T. Jones, M. Torsten, L. Yurramendi, J. Sustain. Metall. 2018 4, 126-146. DOI: 10.1007/s40831-018-0162-8.

[7] S. M. Jowitt, T. T. Werner, Z. Weng, G. M. Mudd, Curr. Opin. Green Sustain. Chem. 2018, 13, 1-7. DOl: 10.1016/j.cog'sc.2018.02.008.

[8] N. Das, D. Das, J. Rare Earths 2013, 31, 933-943. DOl: 10.1016/51002$0721(13) 60009-5$.

[9] S. Pavón, A. Fortuny, M. T. Coll, A. M. Sastre, Waste Manag. 2018, 82 , 241-248. DOI: 10.1016/j.wasman.2018.10.027.

[10] T. G. Ambaye, M. Vaccari, F. D. Castro, S. Prasad, S. Rtimi, Environ. Sci. Pollut. Res. Int. 2020, 27, 36052-36074. DOl: 10.1007/s11356-020-09630-2.

[11] J. A. Kim, G. Dodbiba, Y. Tanimura, K. Mitsuhashi, N. Fukuda, K. Okaya, S. Matsuo, T. Fujita, Mater. Trans. 2011, 52, 1799-1806. DOI: 10.2320/ matertrans.M2011111.

[12] E. Fabre, C. B. Lopes, C. Vale, E. Pereira, C. M. Silva, Sci. Total Environ. 2020, 709, 135883. DOl: 10.1016/j.scitotenv.2019.135883.

[13] M. Dias, J. Pinto, B. Henriques, P. Figueira, E. Fabre, D. Tavares, C. Vale, E. Pereira, Int. J. Environ. Res. Public Health 2021, 18, 1-17. DOI: 10.3390/ ijerph18041580.

[14] B. Henriques, L. S. Rocha, C. B. Lopes, P. Figueira, R. J. R. Monteiro, A. C. Duarte, M. A. Pardal, E. Pereira, Chem. Eng. J. 2015, 281, 759-770. DOl: 10.1016/j.cej.2015.07.013.

[15] J. Sadhukhan, S. Gadkari, E. Martinez-Hernandez, K. S. Ng, M. Shemfe, E. Torres-Garcia, J. Lynch, Green Chem. 2019, 21, 2635-2655. D0I: 10.1039/ c9gc00607a.

[16] J. M. S. C. D. Coimbra, Controlo de Qualidade Aplicado à Análise de Água de Consumo Por ICP-MS, Tese de Mestrado, Universidade de Aveiro, 2007. ria. ua.pt/handle/10773/4862

[17] F. J. L. Gordillo, J. Aguilera, C. Wiencke, C. Jiménez, J. Plant Physiol. 2015, 173, 41-50. DOI: 10.1016/j.jplph.2014.09.008.

[18] E. Fabre, M. Dias, B. Henriques, T. Viana, N. Ferreira, J. Soares, J. Pinto, C. Vale, J. Pinheiro-Torres, C. M. Silva, E. Pereira, Environ. Sci. Pollut. Res. Int. 2021, 28, 30255-30266. DOI: 10.1007/s11356-021-12687-2.

[19] V. Murphy, H. Hughes, P. McLoughlin, J. Hazard. Mater. 2009, 166, 318-326. DOI: 10.1016/j.jhazmat.2008.11.041.

[20] T. D. Pickering, V. H. Sladden, R. H. Furneaux, J. A. Hemmingson, P. Redfearn, J. Appl. Phycol. 1993, 5, 85-91. DOl: 10.1007/BF02182425.

[21] L. Qu, J. Xu, J. Sun, X. Li, K. Gao, Aquaculture 2017, 473, 383-388. DOI: 10.1016/j.aquaculture.2017.03.001.

[22] M. Costa, B. Henriques, J. Pinto, E. Fabre, T. Viana, N. Ferreira, J. Amaral, C. Vale, J. Pinheiro-Torres, E. Pereira, Environ. Pollut. 2020, 266, 115374. DOI: 10.1016/j.envpol.2020.115374.

[23] M. Costa, B. Henriques, J. Pinto, E. Fabre, M. Dias, J. Soares, L. Carvalho, C. Vale, J. Pinheiro-Torres, E. Pereira, Chemosphere 2020, 252, 126562. DOI: 10.1016/j.chemosphere.2020.126562.

[24] J. Pinto, M. Costa, B. Henriques, J. Soares, M. Dias, T. Viana, N. Ferreira, C. Vale, J. Pinheiro-Torres, E. Pereira, J. Rare Earths 2020, 39, 734-741. DOI: 10.1016/j.jre.2020.09.025.

[25] J. Pinto, B. Henriques, J. Soares, M. Costa, M. Dias, E. Fabre, C. B. Lopes, C. Vale, J. Pinheiro-Torres, E. Pereira, J. Environ. Manage. 2020, 263, 110376. DOI: 10.1016/j.jenvman.2020.110376. 


\section{Bruno Henriques}

Departamento de Quími-

ca, Universidade de Aveiro.

LAQV/REQUIMTE - Laboratório Associado para a Química Verde.

Doutorado em Química, é investigador no Departamento de Química da Universidade de Aveiro. A sua área de atividade científica inclui a Química Analítica e Ambiental, e a Biotecnologia, aplicadas à melhoria da qualidade da água, e recuperação de elementos de alto valor económico e/ou estratégico, num contexto de sustentabilidade e economia circular assim como a avaliação da contaminação ambiental e seus impactos na biota. brunogalinho@ua.pt ORCID.org/0000-0002-6472-5479

\section{$>$}

\section{Carlos Vale}

CIIMAR - Centro Interdisciplinar de Investigação Marinha e Ambiental.

Possui habilitação/agregação em oceanografia química, ex-diretor de departamento, diretor do conselho científico e vice-diretor do IPMA, e vice-presidente do CIEM. A sua especialização inclui o comportamento de contaminantes nos ecossistemas marinhos, as interações com os organismos e os impactos dos contaminantes nos sistemas ambientais em evolução. carlos.vale@ciimar.up.pt ORCID.org/0000-0003-0162-1960

\section{$>$}

\section{Daniela Tavares}

Departamento de Química, Universidade de Aveiro. É doutorada em Química pela Universidade de Aveiro e Técnica Superior de Investigação no âmbito do Projeto de Co-promoção N9ve-Rare Earth Elements. A sua investigação tem-se focado no desenvolvimento e aplicação de novos nanomateriais, bem como na aplicação de biossorventes em novas tecnologias para a remediação de águas contaminadas. danielatavares@ua.pt ORCID.org/0000-0002-3196-7922

\section{Eduarda Pereira}

Departamento de Química, Universidade de Aveiro.

LAQV/REQUIMTE - Laboratório Associado para a Química Verde.

É professora associada no Departamento de Química da Universidade de Aveiro e coordenadora do Laboratório Central de Análises da mesma Universidade. Possui larga experiência científica na avaliação da qualidade ambiental e poluição de zonas estuarinas e costeiras, incluindo bioacumulação e bioamplificação de elementos potencialmente tóxicos ao longo de cadeias tróficas estuarinas. eduper@ua.pt ORCID.org/0000-0002-6046-5243

$>$

\section{${ }^{*}$ Filipe L. S. Monteiro}

Departamento de Quími-

ca, Universidade de Aveiro.

É licenciado em Química Analítica pela Universidade de Aveiro. Trabalhou na indústria, com funções na área da qualidade, de direção de produção e desenvolvimento de novos produtos e tecnologias. É divulgador de ciência, realizando sessões de promoção da Química com recurso ao ilusionismo. A investigação centra-se na procura de soluções aplicadas ao Ambiente e recuperação de elementos de alto valor económico e/ou estratégico. filipelsmonteiro@ua.pt ORCID.org/0000-0002-9536-0302

\section{$>$}

\section{Jéssica Jacinto}

Departamento de Quími-

ca, Universidade de Aveiro.

LAQV/REQUIMTE - Laboratório AsSociado para a Química Verde.

É mestre em Biotecnologia Ambiental e Industrial pela Universidade de Aveiro. Atualmente é bolseira no programa doutoral em Química Sustentável, na mesma instituição. 0 trabalho de investigação que desenvolve tem por objetivo criar soluções mais sustentáveis para o fornecimento de matérias-primas críticas que encontram aplicação em tecnologias verdes emergentes. jessicajacinto@ua.pt ORCID.org/0000-0003-0752-9911

\section{João Colónia}

Departamento de Química, Universidade de Aveiro.

É licenciado em Biotecnologia pelo Departamento de Química da Universidade de Aveiro. Atualmente está a finalizar o mestrado em Biotecnologia Industrial e Ambiental, sendo bolseiro de investigação no Departamento de Química. A sua investigação foca-se na recuperação de elementos de elevado interesse tecnoló gico a partir de resíduos eletrónicos com recursos a macroalgas marinhas vivas. joaocolonia@ua.pt ORCID.org/0000-0001-5928-1845

$>$

\section{João Pinto}

Departamento de Química, Universidade de Aveiro. LAQV/REQUIMTE - Laboratório AsSociado para a Química Verde. Mestre em Ciências do Mar e da Atmosfera e aluno de doutoramento em Química no Departamento de Química da Universidade de Aveiro. A sua investigação baseia-se na avaliação de toxicidade de contaminantes emergentes e na sua recuperação através de processos de bioacumulação por parte de macroalgas marinhas. joao.pedro.pinto@ua.pt ORCID.org/0000-0002-6175-2906

\section{$>$}

\section{Nicole Ferreira}

Departamento de Química, Universidade de Aveiro.

É mestre em Química Analítica e Controlo de Qualidade e licenciada em Química pela Universidade de Aveiro, sendo atualmente bolseira de investigação no Projeto em Co-promoção I\&D Empresas intitulado MARemPó. A sua investigação tem como foco a validação de metodologias para quantificação de elementos macro, micro e potencialmente tóxicos em matrizes ambientais e alimentares, além da fortificação de minerais essenciais em macroalgas marinhas. nicoleviana@ua.pt ORCID.org/0000-0001-8150-5227

\section{Thainara Viana}

Departamento de Química, Universidade de Aveiro.

É mestre em Biotecnologia molecular e licenciada em Biotecnologia pela Universidade de Aveiro, sendo bolseira de investigação no Projeto de Co-promoção N9ve-Rare Earth Elements, no Departamento de Química da mesma instituição. A sua investigação baseia-se na biotecnologia ambiental e molecular aplicada à remoção e recuperação de elementos de alto valor económico, como terras raras, através da utilização de macroalgas vivas. thainara@ua.pt ORCID.org/0000-0001-5246-4809 